\title{
Reduced neutron widths in the nuclear data ensemble: Experiment and theory do not agree
}

\author{
P. E. Koehler, a \\ Physics Division, Oak Ridge National Laboratory,Oak Ridge, TN 37831
}

\begin{abstract}
I have analyzed reduced neutron widths $\left(\Gamma_{n}^{0}\right)$ for the subset of 1245 resonances in the nuclear data ensemble (NDE) for which they have been reported. Random matrix theory (RMT) predicts for the Gaussian orthogonal ensemble (GOE) that these widths should follow a $\chi^{2}$ distribution having one degree of freedom $(v=1)$ - the Porter Thomas (PT) distribution. Using the maximum-likelihood (ML) technique, I have determined that the $\Gamma_{n}^{0}$ values in the NDE are best described by a $\chi^{2}$ distribution having $v=0.801 \pm 0.052$, which is 3.8 standard deviations smaller than predicted by RMT. I show that this striking disagreement is most likely due to the inclusion of significant $p$-wave contamination to the supposedly pure $s$-wave NDE. Furthermore, when an energy-dependent threshold is used to remove the $p$-wave contamination, ML analysis yields $v=1.217 \pm 0.092$ for the remaining data, still in poor agreement with the RMT prediction for the GOE. These results cast very serious doubt on claims that the NDE represents a striking confirmation of RMT.
\end{abstract}

\section{Introduction}

The nuclear data ensemble (NDE) [1,2] is a set of 1407 resonance energies consisting of 30 sequences in 27 different nuclides. The ensemble was assembled to test predictions of random matrix theory (RMT) [3]. Fluctuation properties of resonance energies in the NDE were found to be in remarkably close agreement with RMT predictions for the Gaussian orthogonal ensemble (GOE). Hence, the NDE often is cited as providing striking confirmation of RMT predictions for the GOE.

Reduced neutron widths $\left(\Gamma_{n}^{0}\right)$ have been reported for a subset of 1245 resonances in the NDE, consisting of 14 to 178 measurements for 24 nuclides, as given in Table 1 . Note that the number of resonances for the $\mathrm{Gd}$ isotopes in Ref. [2] are incorrect, and that one resonance for ${ }^{182} \mathrm{~W}$ (at $1920.9 \mathrm{eV}$ ) does not have a measured neutron width, and so there are only 40 instead of 41 resonances for this nuclide included in my analyses.

If the GOE correctly describes the data, RMT predicts these widths should follow a $\chi^{2}$ distribution having one degree of freedom $(v=1)$ - the Porter Thomas (PT) distribution. In Ref. [2] the maximum-likelihood (ML) technique was used to obtain the most likely value of $v$ for a subset of the NDE data and it was concluded that there was satisfactory accord between theory and experiment. However, there are several problems with the analysis of Ref. [2], which I will describe below. I find that when the data are analyzed more carefully, they do not agree with RMT predictions for the GOE.

For the remained of this paper when referring to the NDE data, I will include the spin statistical factor $g=$ $\frac{2 J+1}{(2 I+1)(2 j+1)}$ (where $J, I$, and $j$ are spins of the resonance,

\footnotetext{
a e-mail: koehlerpe@ornl.gov
}

Table 1. NDE nuclides.

\begin{tabular}{|c|c|c|c|c|}
\hline Nuclide & $\mathrm{N}_{r e s}$ & $\mathrm{E}_{\text {max }}(\mathrm{keV})$ & $v_{\min }$ & $v_{p f}$ \\
\hline${ }^{64} \mathrm{Zn}$ & 103 & 367.55 & $1.35^{+0.24}$ & $1.54^{+0.29}$ \\
\hline${ }^{66} \mathrm{Zn}$ & 65 & 297.63 & $0.68^{+0.23}$ & $0.74^{-0.26}$ \\
\hline${ }^{68} \mathrm{Zn}$ & 45 & 247.20 & $0.75^{-0.21}+0.27$ & $0.95^{-0.36}$ \\
\hline${ }^{114} \mathrm{Cd}$ & 17 & 3.3336 & $0.35_{-0.34}^{-0.55}$ & $2.0_{-12}^{+0.52}$ \\
\hline${ }^{152} \mathrm{Sm}$ & 70 & 3.665 & $1.14_{-0.25}^{+0.234}$ & $1.55_{-0.38}^{+0.2}$ \\
\hline${ }^{154} \mathrm{Sm}$ & 27 & 3.0468 & $0.76_{-0.38}^{+0.25}$ & $\begin{array}{l}-0.38 \\
1.32_{-0}^{+0.65}\end{array}$ \\
\hline${ }^{154} \mathrm{Gd}$ & 19 & 0.2692 & $0.44_{-0.43}^{+0.36}$ & $0.49_{-0.48}^{+0.63}$ \\
\hline${ }^{156} \mathrm{Gd}$ & 54 & 1.9908 & $1.22^{+0.27}+0.27$ & $1.44^{+0.51}$ \\
\hline${ }^{158} \mathrm{Gd}$ & 47 & 3.9827 & $0.75^{-0.26}+0.25$ & $1.17^{-0.49}+0.54$ \\
\hline${ }^{160} \mathrm{Gd}$ & 21 & 3.9316 & $0.55^{-0.22}+0.34$ & $0.83^{-0.47}$ \\
\hline${ }^{160} \mathrm{Dy}$ & 18 & 0.4301 & $0.83^{-0.33}$ & $\begin{array}{l}0.05^{-0.65} \\
1.41^{+1.00}\end{array}$ \\
\hline${ }^{162}$ Dy & 46 & 2.9572 & $1.02_{-0.33}^{+0.53}$ & $0.99^{-0.83}+0.47$ \\
\hline${ }^{164}$ Dy & 20 & 2.9687 & $0.82_{-0.44}^{+0.52}$ & $\begin{array}{c}-0.43 \\
2.3_{-1 .}^{+1.2}\end{array}$ \\
\hline${ }^{166} \mathrm{Er}$ & 109 & 4.1693 & $0.85_{-0.18}^{-0.44}$ & $1.85_{-0.49}^{+0.0}$ \\
\hline${ }^{168} \mathrm{Er}$ & 48 & 4.6711 & $0.80_{-0.30}^{+0.11}$ & $1.32_{-0.65}^{+0.45}$ \\
\hline${ }^{170} \mathrm{Er}$ & 31 & 4.7151 & $0.36_{-0.34}^{+0.27}$ & $3.6_{-1 .}^{+1.65}$ \\
\hline${ }^{172} \mathrm{Yb}$ & 55 & 3.9000 & $0.71^{-0.31}+0.27$ & $0.70^{-1.3}+0.30$ \\
\hline${ }^{174} \mathrm{Yb}$ & 19 & 3.2877 & $0.80^{+0.44}$ & $1.29^{+0.26}+0.68$ \\
\hline${ }^{176} \mathrm{Yb}$ & 23 & 3.9723 & $0.04_{-0.03}^{+0.29}+{ }^{+0.29}$ & $1.05_{-0.55}^{-0.58}+0.55$ \\
\hline${ }^{182} \mathrm{~W}$ & 40 & 2.6071 & $0.76_{-0.35}^{-0.03}$ & $1.50_{-0.52}^{-0.55}$ \\
\hline${ }^{184} \mathrm{~W}$ & 30 & 2.6208 & $0.62_{-0.34}^{+0.35}$ & $0.99_{-0.48}^{-0.55}$ \\
\hline${ }^{186} \mathrm{~W}$ & 14 & 1.1871 & $1.23^{-0.31}$ & $1.32^{-0.48}+0.93$ \\
\hline${ }^{232} \mathrm{Th}$ & 178 & 2.988 & $0.76_{-0.13}^{+0.62}$ & $1.78^{+0.36}$ \\
\hline${ }^{238} \mathrm{U}$ & 146 & 3.0151 & $0.79 \pm 0.12$ & $1.02^{+0.39}$ \\
\hline W.A. & 1245 & - & $0.801 \pm 0.052$ & $1.217 \pm 0.092$ \\
\hline
\end{tabular}

target, and neutron, respectively) in the definition of the reduced neutron width, replacing $\Gamma_{n}^{0}$ with $g \Gamma_{n}^{0}$. Although all the target nuclides considered have $I=0$, and hence $J=1 / 2$ and $g=1$ for $s$-wave resonances, I will show below that the NDE almost certainly suffers from signifi-

This is an Open Access article distributed under the terms of the Creative Commons Attribution-Noncommercial License, which permits unrestricted use, distribution, and reproduction in any noncommercial medium, provided the original work is properly cited. 
cant $p$-wave contamination. There are two resonance spins possible for $p$-wave resonances for these nuclides, $J=1 / 2$ or $3 / 2$, and hence $g=1$ or 2 , which remain undetermined. In these cases, the reported neutron widths are essentially $g \Gamma_{n}$, and $g \Gamma_{n}^{0}=g \Gamma_{n} / \sqrt{E_{n}}$ are "effective" reduced neutron widths rather that the true reduced neutron widths for $p$ wave resonances.

\section{Importance of threshold in ML analysis of neutron widths}

To perform a meaningful ML analysis, the data should be complete (no missing resonances) and pure (all resonances have the same parity), or the analysis technique must take into account the incompleteness of the data, and at least attempt to assess the purity of the data. Along these lines, there are at least four important limitations of experiments from which the NDE data were obtained that must be taken into account.

First, every experiment has a finite threshold below which widths are not observed. Therefore, even if a pure $s$-wave sequence of resonances could be obtained from a measurement, it will very likely not be complete. Hence, the necessity of using a threshold in the ML analysis to obtain $v$ from the data was realized from the beginning [4] of such analyses.

A second important limitation is that all experiments have a threshold below which they cannot distinguish $s$ from $p$-wave resonances. Therefore, unless some independent means exists for reliably separating resonances of opposite parity, a threshold may again be needed to ensure that the data are all of the same parity [5].

A third consideration related to threshold may also be important when analyzing a set of widths comprised of many subsets from different nuclides: Each experiment from which the data were obtained may have had a different threshold. Therefore, if the data are analyzed using a single threshold, it must be at least as high as that for the nuclide having the highest threshold. In Ref. [2], a subset of 1182 widths from the NDE was analyzed using a common energy-independent threshold. In Fig. 1, minimum reduced neutron widths $\left(g \Gamma_{n, \min }^{0}\right)$, normalized to their respective average reduced neutron widths $\left.\left(<g \Gamma_{n}^{0}\right\rangle\right)$ are shown for the NDE nuclides. As can be seen, the individual data sets appear to have widely varying sensitivities. Hence, using a common threshold in the ML analysis could lead to a systematic error in the returned value of $v$, unless a threshold at least as high as the maximum $g \Gamma_{n, \min }^{0} /<g \Gamma_{n}^{0}>$ was used. However, using a threshold this high on the entire data set will exclude much of the data from the analysis, and hence will result in reduced precision.

The fourth potential problem is that the experimental thresholds are energy dependent. This fact is evident in Fig. 2 in which all 1245 neutron widths in the NDE are shown as a function of resonance energy. In this figure, it can be seen that there are fewer small widths observed as the energy increases. Causes for this effect are explained in Ref. [6]. Therefore, if an energy-independent threshold is

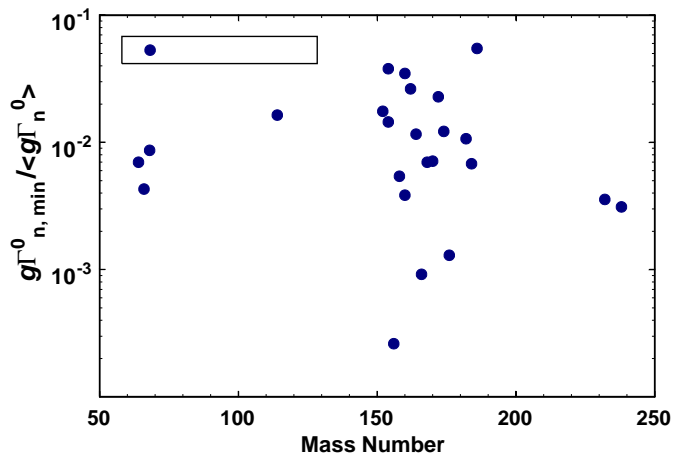

Fig. 1. Minimum reduced neutron widths, normalized to the respective averages, for each of the nuclides in the NDE, versus mass number.

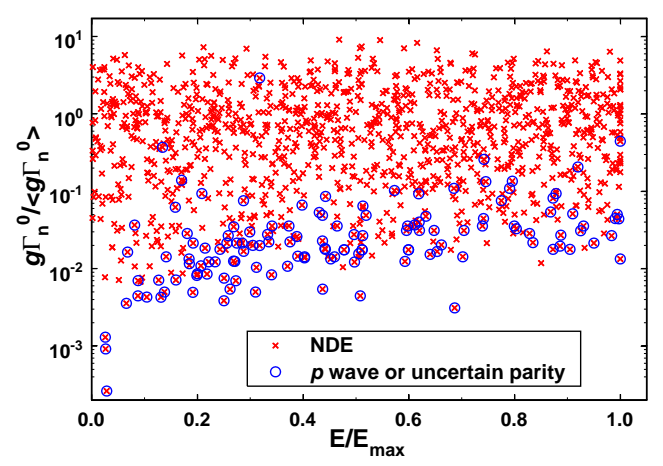

Fig. 2. All 1245 reduced neutron widths in the NDE (X's). Data for each nuclide have been normalized to their respective average reduced neutron widths and maximum energies. Circles depict those resonances which have been identified as being $p$ wave or of uncertain parity.

used, as is the case in all previous ML analyses of which I am aware, it must be at least as high as the threshold at the maximum energy. However, using such a high threshold will exclude much of the data and result in reduced statistical precision.

The effect of a measurement threshold on a pure set of $s$-wave resonances, and the systematic error caused by assuming all resonances have been observed are illustrated in Fig. 3. Integral plots of $\chi^{2}$ distributions having $v=0.5$, 1.0 , and 2.0 are shown in the top part of this figure. As can be seen, the distribution narrows as $v$ increases. The middle part of this figure shows a $v=1$ distribution before and after a threshold proportional to resonance energy (e.g., $\Gamma_{n}^{0} \geqq a E_{n}$, where $a$ is a constant) has been applied, resulting in the loss of some small widths. The bottom part of this figure shows the same incomplete $v=1$ distribution, after it has been renormalized assuming that all widths had been observed. It is compared to the same $v=1$ and 2 dis- 


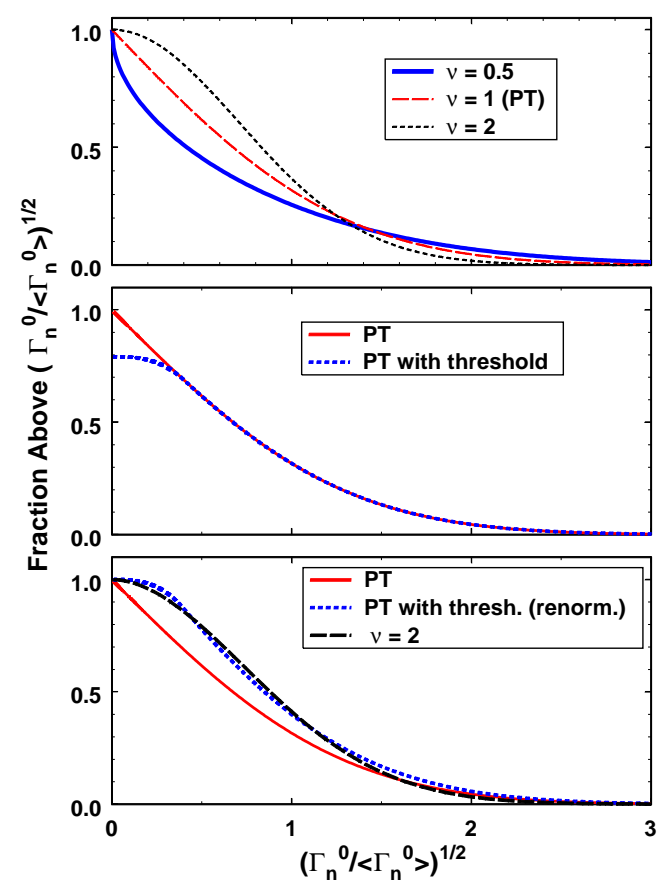

Fig. 3. Top: Three $\chi^{2}$ distributions with $v=0.5,1$, and 2. Plotted are the fraction of widths greater than the square root of a given width versus the square root of the width (normalized to the average width). Middle: Porter-Thomas (PT) distributions before (solid curve) and after (dashed curve) application of a threshold of the form $\Gamma_{n}^{0} \geqq a E_{n}$, where $a$ is a constant. This threshold causes small widths to be excluded. Bottom: The solid curve is the same PT distribution as in the top two panels. The dashed curve is the same dashed curve as in the middle panel, after being renormalized assuming all widths had been observed. The long-dashed curve is a $\chi^{2}$ distribution for $v=2$. See text for details.

tributions in the top part of the figure. As can be seen, the renormalized "data" agree much better with the $v=2$ distribution, hence illustrating the general result that using no or too low an analysis threshold in an ML analysis of a data set with missing small widths will tend to result in a falsely large value of $v$. On the other hand, using too low an analysis threshold can, in certain circumstances, have the opposite effect. For example, because $p$-wave resonances have, on average, much smaller neutron widths, including a few $p$-wave resonances in an otherwise pure and complete $s$-wave set will tend to result in a falsely small value of $v$. In addition, for such a $p$-wave-contaminated set, the value of $v$ returned from a ML analysis will tend to systematically increase as the analysis threshold is raised.

To minimize the effects of the above problems I have done separate ML analyses for each nuclide in the NDE using separate energy-dependent thresholds. A weighted average of these results is then compared to theory, as explained in the next section.

\section{An improved ML analysis of the NDE neutron widths}

The PT distribution is a special case of the class of $\chi^{2}$ distributions:

$$
P(y, v, \bar{y}) d y=\frac{v}{2 G\left(\frac{v}{2}\right)}\left(\frac{v y}{2 \bar{y}}\right)^{\frac{v}{2}-1} \exp \left(-\frac{v y}{2 \bar{y}}\right) d y,
$$

where $P(y, v, \bar{y})$ is the probability, $v$ the degrees of freedom, $y$ the reduced neutron width $g \Gamma_{n}^{0}, \bar{y}$ the average reduced width $<g \Gamma_{n}^{0}>$, and $G\left(\frac{v}{2}\right)$ is the gamma function for $\frac{v}{2}$. For the PT distribution, $v=1$. As was done in Refs. [2,5], I assumed that reduced neutron widths were distributed according to Eq. 1 and the maximum-likelihood technique was used to determine the most likely values of $v$ and $\left\langle g \Gamma_{n}^{0}>\right.$. However, as discussed above, the method was improved by allowing the threshold to be energy dependent. Therefore, the probability distribution considered was

$$
F\left(y, y_{t}, v, \bar{y}\right) d y=\frac{P(y, v, \bar{y}) d y}{\int_{E_{1}}^{E_{2}} \int_{y_{t}}^{\infty} P(y, v, \bar{y}) d y d E}
$$

where $E_{1}$ and $E_{2}$ are the lower and upper neutron energy limits of the data used, and the threshold on $g \Gamma_{n}^{0}$ is defined by $y_{t}=a E^{b}$, with $a$ and $b$ being constants. The likelihood function for $n$ widths $y_{1}, \ldots ., y_{n}$ was constructed

$$
L\left(y_{t}, v, \bar{y}\right)=\prod_{i=1}^{n} F\left(y_{i}, y_{t}, v, \bar{y}\right)
$$

and evaluated on a grid of $v$ and $\bar{y}$, and the maximum found.

Uncertainties in $v$ and $\left\langle g \Gamma_{n}^{0}>\right.$, due to finite sampling errors, typically [5] are determined by assuming that each parameter (for a given value of the other parameter) is Gaussian distributed about the optimum value; hence, it follows that the variance in $v$ is given by $\sigma_{v}^{2}=-\left[\delta^{2} L / \delta v^{2}\right]^{-1}$. However, I found that this procedure tended to underestimate the uncertainty in $v$, in the direction of increasing $v$, when $v$ was substantially smaller than one. Therefore, I employed an alternative, more conservative method. Curves of $L$ as functions of $v$ were calculated in both directions from the optimum value. For each value of $v,<g \Gamma_{n}^{0}>$ was allowed to vary until the maximum $L$ was found. These curves were found to be very close to Gaussian in shape, and hence two values of $\sigma_{v}$ were calculated from the points in each direction where the function was $e^{-1 / 2}$ of its maximum. In all cases, the smaller of these two uncertainties was at least as large as that calculated using the standard technique. When calculating the weighted average for the NDE, I used variances in the direction towards $v=1$ as weights.

Thresholds (on $g \Gamma_{n}^{0}$ ) were of the form $y_{t}=a E$ (i.e. $b=1$ ) for two reasons. First, this form appeared to reproduce the energy dependence of the minimum widths for most nuclides in the NDE. Second, this form eliminates, to good approximation, $p$-wave contamination equally effectively at all energies. This is because the penetrability factors for $s$ - and $p$-wave resonances vary as $E^{1 / 2}$ and 

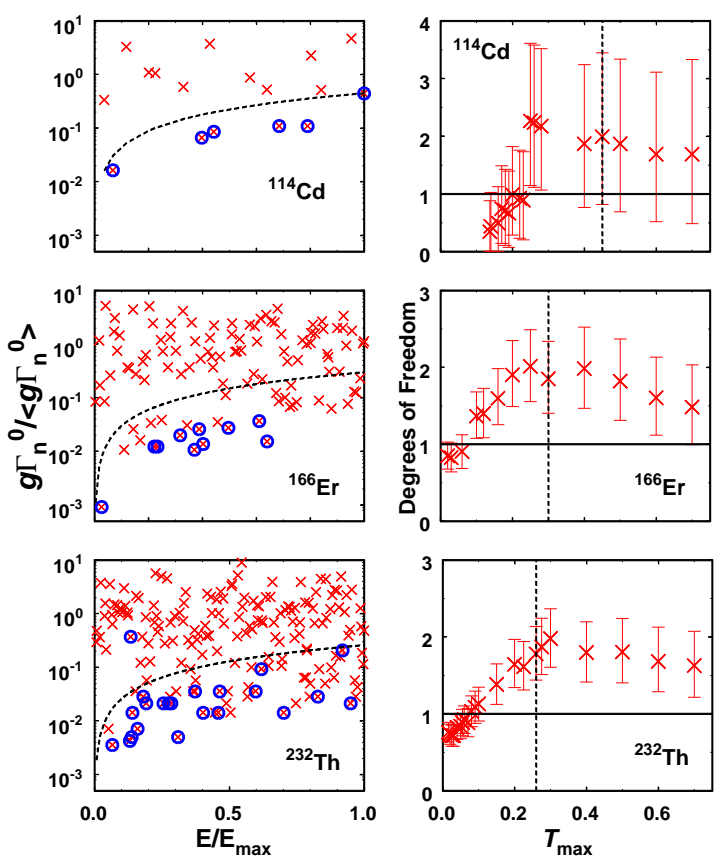

Fig. 4. Left: normalized reduced neutron widths versus normalized resonance energies for ${ }^{114} \mathrm{Cd},{ }^{166} \mathrm{Er}$, and ${ }^{232} \mathrm{Th}$ resonances in the NDE. X's depict all resonances in the NDE whereas circles show resonances previously identified as being $p$ wave or of uncertain parity. Right: $v$ values from ML analyses versus thresholds used, for the same three nuclides. Error bars correspond to one-standard-deviation uncertainties. Dashed vertical lines correspond to thresholds depicted by dashed curves in the left part of this figure. See text for details.

(approximately) $E^{3 / 2}$, respectively. Therefore, because the data had already been converted to reduced neutron widths, by dividing the widths by $E^{1 / 2}$ (i.e., assuming they were all $s$ wave), any remaining $p$-wave resonances will retain an underlying energy dependence proportional to $E$. The reason for excluding $p$-wave resonances will become apparent below.

Results of the maximum-likelihood analyses for three NDE nuclides are shown in Fig. 4. On the left of this figure, reduced neutron widths (normalized to their respective average values) are plotted as functions of resonance energy (normalized to the maximum resonance energy, $E_{\text {max }}$, used in each case). On the right side of this figure, $v$ values from the maximum-likelihood analysis are plotted versus the threshold coefficient $T_{\max }$, where $T_{\max }$ corresponds to the threshold value of $g \Gamma_{n}^{0} /<g \Gamma_{n}^{0}>$ at the maximum energy. In other words, thresholds can be expressed as

$$
\frac{g \Gamma_{n}^{0}}{<g \Gamma_{n}^{0}>} \geqq T_{\max } E / E_{\max } .
$$

For the initial analysis, thresholds just below the smallest observed resonance (in terms of Eq. 4) for each nuclide were used; hence, it was implicitly assumed that all res- onances above these threshold curves were observed. Because experiment thresholds might not be precisely sharp, such an assumption might be expected to result in $v$ values which are systematically a bit large. However, the weighted average of the results for the 24 nuclides in the NDE is $v=0.801 \pm 0.052$, which is 3.8 standard deviations smaller than the expected result of $v=1$ for the GOE. Degrees-offreedom values for each of the NDE nuclides at these minimum thresholds are given in column four $\left(v_{\min }\right)$ of Table 1 . According to Ref. [7], transition strength distributions are expected to become gradually wider ( $v$ gradually decreasing from 1) as the system becomes more regular. Hence, the result $v=0.801 \pm 0.052$ could be interpreted as evidence that the nuclides in the NDE are more regular than chaotic. A more likely explanation is that the NDE contains sizeable $p$-wave contamination. In either case, this result for $v$ casts serious doubt on claims $[1,2]$ that the NDE represents a striking confirmation of RMT predictions for the GOE.

\section{Cleansing the NDE of $p$-waves}

That the NDE is contaminated by $p$-wave resonances is evident in Figs. 2 and 4. In these figures, resonances in the NDE that have been identified (in Refs. [8] and [9] and references contained therein) as $p$-wave or of uncertain parity are shown as open circles. That many of these resonances are in fact $p$-wave is reinforced by the behaviour of the $v$ values from the ML analyses as functions of threshold, as shown in the right side of Fig. 4. In all three cases shown, $v$ steadily increases at the lower thresholds and then gradually stabilizes at higher thresholds. This is just the behaviour expected for a population of $s$-wave resonances contaminated by $p$-wave resonances. Similar fractions of previously identified $p$-wave resonances and trends in $v$ with threshold are seen for several of the other NDE nuclides. Hence, it is fairly certain that the NDE suffers from significant $p$-wave contamination.

Removing effects of these $p$-wave resonances from the NDE ML analysis is a simple matter of raising thresholds until they are above the largest previously-identified $p$-wave resonance and/or $v$ stabilizes as a function of threshold. When this is done, the resulting weighted average is still in conflict with the RMT prediction for the GOE, albeit in the opposite direction from the result using the lowest thresholds: $v=1.217 \pm 0.092$ (2.4 standard deviations larger than PT). Typical " $p$-wave free" thresholds for three NDE nuclides are shown as dashed curves in the left-hand part of Fig. 4. Degrees-of-freedom values for each of the NDE nuclides at these " $p$-wave free" thresholds are given in column five $\left(v_{p f}\right)$ of Table 1.

\section{Why does the NDE agree so well with spacing statistics?}

Given the above result that reduced neutron widths in the NDE do not agree with the GOE width distribution, and 
the fact that this data set contains many resonances that have been assigned as being definitely $p$ wave using reliable techniques (e.g., [10]), it is natural to ask why the same data agree so well with all the GOE spacing statistics examined in Refs. [1,2]. The answer, I think, has to do with how the NDE data were selected. For example, in Ref. [2] it is stated that "The criterion for inclusion in the NDE is that the individual sequences be in general agreement with the GOE."

Data for all but three of the 24 nuclides considered herein were obtained by the group at Columbia University. According to their publications (e.g., Ref.[11]), they had "...no specific tests for $s$ vs $p$ levels, so there may be errors in these assignments." Therefore, they relied on theoretical guidance, specifically measures derived from the $\mathrm{GOE}$, to perform these separations. For example, for six of the 24 nuclides considered herein, including the two having the largest number of resonances, separation of $p$ - from $s$-wave resonances was accomplished [12] by first calculating the overall number of $s$-wave resonances by assuming PT was correct. Then, which small resonances to assign to the $s$-wave set was determined by requiring good agreement with four spacing statistics (Wigner, $\rho\left(S_{j}, S_{j+1}\right), \Delta_{3}$, and the Dyson $F$ test) related to the GOE. Furthermore, for several other NDE nuclides, PT again was assumed to be correct and used to calculate the total number of $s$-wave resonances. Then, a Bayesian analysis was used to decide which of the small resonances to assign to the $s$-wave set. Such Bayesian analyses are known to be unreliable. For example, several neutron resonances in ${ }^{64} \mathrm{Zn}$ [13] are known to be definitely $p$ wave by their symmetrical shape in transmission (total cross section) data, but nevertheless have a Bayesian probability of $>99 \%$ of being $s$ wave.

\section{Hope for better data in the future}

The main problem in obtaining neutron data suitable for stringent tests of RMT is reliably separating small $s$ - from large $p$-wave resonances. Of the data in the NDE, only for ${ }^{64,66,68} \mathrm{Zn}$ [13-15] has this been done using a method independent of the theory being tested. In these cases, the shape of the resonances in the transmission data was used; $s$-wave resonances have a characteristic asymmetric shape due to interference with the comparatively large $s$-wave potential scattering. However, this method fails for resonances having small neutron widths and, in fact, such resonances may not even be visible in the transmission data.

A solution to this problem can be found in better neutroncapture measurements. It has been known for many years [16] that information contained in the $\gamma$-ray cascade following neutron capture can be used to determine resonance spins and parities. Relatively few such measurements have been made however, mainly due to increased difficulty, and unsuitability of the apparatus for obtaining absolute cross sections. However, using a new technique, it recently has been demonstrated [17] that resonance spin measurements are relatively easy using a $4 \pi \mathrm{BaF}_{2}$ detector at a white neutron source. Even more recently [18] it has been shown that a much more modest detector, when coupled to a highresolution white neutron source, can obtain even better results; determining both resonance spins and parities. These new developments hold the promise of solving the perennial problem of separating small $s$ - from large $p$-wave resonances.

\section{Other reported deviations from PT}

There have been several other reported deviations of reducedneutron-width data from the expected PT distribution, which have for the most part been ignored. Most recently, in Ref. [17] it was demonstrated that the $\Gamma_{n}^{0}$ distribution for $s$-wave resonances in ${ }^{147} \mathrm{Sm}$ changes from being consistent with PT $(v=0.91 \pm 0.32)$ for the $E_{n}<350 \mathrm{eV}$ region, to inconsistent with PT $(v=3.19 \pm 0.83)$ for the $350<E_{n}<700$ $\mathrm{eV}$ region.

Similar deviations from a PT distribution have been reported for ${ }^{232} \mathrm{Th}$ [19-22]. However, as shown in Fig. 5 the energy dependence is just the opposite: The data disagree with PT at the lowest energies, but are in agreement at higher energies. The ${ }^{232} \mathrm{Th}$ NDE data, above a threshold of the form given by Equation 4 with $T_{\max }=0.26$ (the same threshold depicted by the dashed curve in the bottom left part of Fig. 4) are shown as solid staircase plots in both panels of the figure. The energy ranges for the data in the top and bottom panels of Fig. 5 were restricted to the first and second $25{ }^{232} \mathrm{Th}$ resonances in the NDE, respectively. The data have been normalized assuming all resonances above threshold have been observed (as implied by Equation 2) with the normalization calculated using the optimum $v$ value from the ML analysis (which correspond the the long-dashed curves). The short-dashed curves correspond to the best $\mathrm{PT}$ distributions according to the ML analysis. In other words, the PT curves were calculated using the $g \Gamma_{n}^{0} /<g \Gamma_{n}^{0}>$ value resulting in the largest $L$ (see Equation 3 ) for $v=1$. The PT curves were normalized to the data, once again assuming that all widths above threshold had been observed. The theory curves in both panels have been adjusted for the effect of the threshold, in the same manner as was done for the middle panel of Fig. 3. Using the ML technique described above, I find that the $v$ value changes from $3.8 \pm 1.3$ for the lower-energy set of resonances to $0.83 \pm 0.68$ for the higher-energy set, thus verifying the claims of Refs. [19-21] that the data are inconsistent with PT at the lower energies and the claim of Ref. [22] that the shape changes back to being consistent with PT at the higher energies.

Finally, in Ref. [23] data for five odd-A nuclides $\left({ }^{151} \mathrm{Sm}\right.$, ${ }^{163} \mathrm{Dy},{ }^{167} \mathrm{Er},{ }^{175} \mathrm{Lu}$, and ${ }^{177} \mathrm{Hf}$ ) were found to disagree with PT despite the fact that the $\Delta_{3}$ statistic indicated that very few resonances had been missed. In all the above cases $v$ was found to be significantly larger than the PT value of 1.0 , just as I have shown it is for the NDE when $p$-wave contamination is eliminated. As far as I know, no explanation has ever been published for these observations.

Given the new and improved experimental techniques of the last few years, it is now possible to obtain data of much better quality than that in the NDE. Hence, in the 


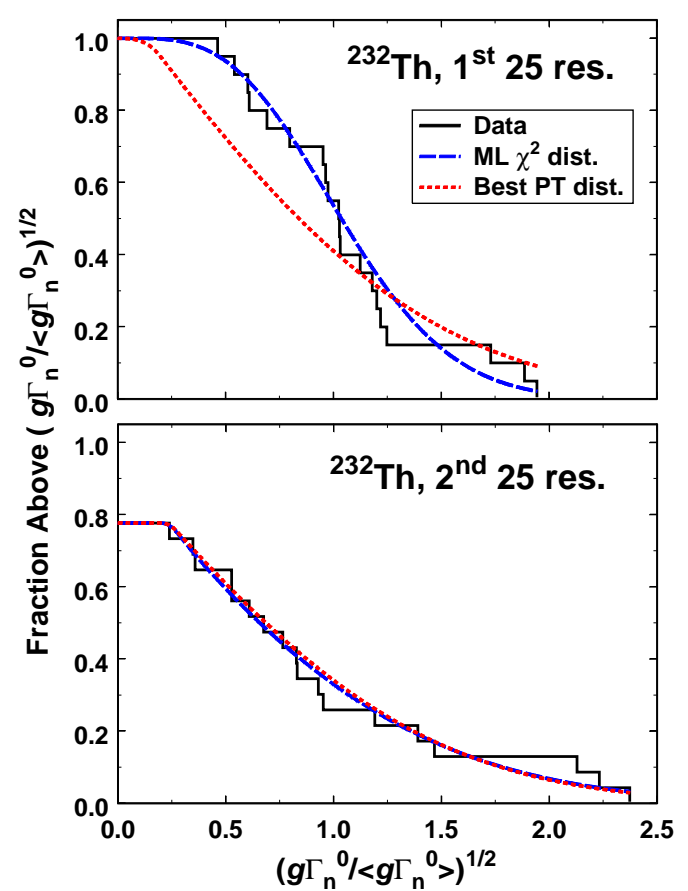

Fig. 5. Top: Observed reduced-neutron-width distribution for the energy range corresponding to the first $25 \mathrm{NDE}$ resonances for ${ }^{232} \mathrm{Th}$. Bottom: Same for second set of 25 resonances. In both panels, solid staircase plots depict the data, long-dashed curves $\chi^{2}$ distributions corresponding to the optimum parameters found in the ML analysis, and short-dashed curves PT distributions in best agreement with the data. A threshold corresponding to the dashed curve in the bottom left panel of Fig. 4 (i.e. $T_{\max }=0.26$ ) was used in the analysis. See text for details.

near future enough data may be available to better elucidate the causes of the observed deviations. However, rarely are new neutron data used to test RMT for the GOE these days. Instead, it is almost always assumed that this theory is correct, and then use it to apply corrections to the data. Therefore, I urge experimentalists obtaining new and improved data to use it, when possible, to test theory.

I would like to thank J. Shriner and J. A. Harvey for fruitful discussions. This work was supported by the U.S. Department of Energy under Contract No. DE-AC05-00OR22725 with UT-Battelle, LLC.

\section{References}

1. R. U. Haq, A. Pandey, and O. Bohigas, Phys. Rev. Lett. 48, (1982) 1086.

2. O. Bohigas, R. U. Haq, and A. Pandey, in Nuclear Data for Science and Technology, edited by K. H. Bockhoff (D. Reidel, Dodrecht, 1983), p. 809.

3. H. A. Weidenmuller and G. E. Mitchell, Reviews of Modern Physics, 81, (2009) 539.

4. C. E. Porter and R. G. Thomas, Phys. Rev. 104, (1956) 483.
5. H. A. Camarda, Phys. Ref. C 49, (1994) 1391.

6. T. Fuketa and J. A. Harvey, Nucl. Instrum. Methods 33, (1965) 107.

7. Y. Alhassid and M. Feingold, Phys. Rev. A 39, (1989) 374.

8. S. F. Mughabghab, Atlas of Neutron Resonances (Elsevier, Amsterdam 2006).

9. S. I. Sukhoruchkin, Z. N. Soroko, and V. V. Deriglazov, Low Energy Neutron Physics (Springer-Verlag, Berlin, 1998).

10. R. Corvi, G. Pasquariello, and T. Van der Veen, in Neutron Physics and Nuclear Data for Reactors and other Applied Purposes, edited by C. Kousnetzoff (Organisation for Economic Co-operation and Development, Paris, 1978) p. 712.

11. H. I. Liou et al., Phys. Rev. C 5, (1972) 974.

12. H. I. Liou, H. S. Camarda, and F. Rahn, Phys. Rev. C 5, (1972) 1002.

13. J. B. Garg, V. K. Tikku, and J. A. Harvey, Phys. Rev. C 23, (1981) 671.

14. J. B. Garg et al., Phys. Rev. C 24, (1981) 1922.

15. J. B. Garg et al., Phys. Rev. C 25, (1982) 1808.

16. C. Coceva et al., Nucl. Phys. A117, (1968) 586.

17. P. E. Koehler et al., Phys. Rev. C 76, (2007) 025804.

18. P. E. Koehler et al., in Capture Gamma-Ray Spectroscopy and Related Topics, edited by A. Blazhev, J. Jolie, N. Warr, and A. Zilges (American Institute of Physics, Melville, New York, 2009) p. 424.

19. P. Ribon, Ph.D. thesis, Universite de Paris, 1969.

20. L. Forman, et al., in Proceedings of the Third Conference on Neutron Cross Sections and Technology, edited by J. A. Harvey and R. L. Macklin (National Technical Information Service, U. S. Dept. of Commerce, Springfield, Virginia, 1971), p. 735.

21. L. Forman et al., Phys. Rev. Lett. 27, (1971) 117.

22. F. Rahn et al., Phys. Rev. C 6, (1972) 1854.

23. H. A. Camarda, Phys. Rev. C 13, (1976) 2524.

24. M. S. Hussein and M. P. Pato, Phys. Rev. Lett. 84, (2000) 3783. 